\title{
Abdominal Compartment Syndrome in Surgical Patients
}

\author{
Alex Muturi ${ }^{1}$ Daniel Ojuka ${ }^{1}$ Peter Ndaguatha ${ }^{1}$, Andrew Kibet ${ }^{2}$ \\ 1. The University Of Nairobi \\ 2. Kenyatta National Hospital
}

Correspondence to: Dr. Alex Muturi, P.O Box 14523-00800 Nairobi, Kenya. Email: almuturi@gmail.com

\begin{abstract}
Background: The deleterious effects of intraabdominal hypertension and abdominal compartment syndrome, affect almost every system. Patients at risk are the critically ill, in whom it leads to altered organ perfusion and end organ dysfunction/failure. The five cases reported highlight the diagnostic and management challenges.

Case Presentation: Five patients with variable degrees of multiple organ dysfunction/failure as evidenced by derangements in laboratory and clinical parameters are presented. Non-surgical interventions including insertion or repositioning of nasogastric tube, insertion of flatus tube, careful titration of IV fluid requirements and appropriate adjustments of ventilator setting were instituted. All five did not respond to initial management and decompressive
\end{abstract}

\section{Introduction}

If the abdominal cavity is treated as a fluid compartment, the pressure within it obeys Pascal's hydrostatic law: when pressure is applied to a contained fluid, the force is transmitted equally in all directions. In this regard, pressure measured at any point within the cavity at any given time can be taken to represent Intra-abdominal pressure (IAP) in the entire abdomen (1).

Intra-abdominal hypertension (IAH) refers to a sustained or repeated pathologic elevation in IAP $\geq 12 \mathrm{~mm} \mathrm{Hg}$ while Abdominal compartment syndrome(ACS) is defined as a sustained IAP over $20 \mathrm{~mm} \mathrm{Hg}$ (with or without Abdominal perfusion pressure(APP) $<60 \mathrm{mmHg}$ ) that is associated with new organ dysfunction/failure $(1,2)$.

IAH occurs when tissue fluid (edema, retroperitoneal blood, free fluid in the abdomen and excessive gas within the intestines) within the peritoneal and laparotomy or re-opening of the abdomen was planned.

Conclusion: Abdominal compartment syndrome can be prevented by regular measurement of intraabdominal pressure in patients at risk. Non-surgical means should precede decompressive laparotomy but timely surgical intervention is crucial.

Key words: case series, Intra-abdominal pressure, Intra-abdominal hypertension, Abdominal compartment syndrome.

Ann Afr Surg. 2017;14(1): 48-52.

DOI: http://dx.doi.org/10.4314/aas.v14i1.10

(C) 2017 Annals of African Surgery. This work is licensed under the Creative Commons Attribution 4.0 International License.

retroperitoneal space accumulate to such a high level that the abdominal wall compliance threshold is exceeded and the abdomen can no longer stretch, at which point continued accumulation results in very high pressure within this compartment (2). The harmful effects of IAH occur long before the manifestation of compartment syndrome (3). When this is not recognized and promptly addressed, it leads to ACS.

In most cases, the underlying pathological process is capillary permeability caused by the systemic inflammatory response syndrome (SIRS) that is the common denominator in critically ill patients. Fluid leaks into the gut wall, mesentery and retroperitoneal tissue. This may be aggravated by overzealous intravenous fluid resuscitation $(2,3)$.

Originally thought to be a disease solely of trauma, IAH and ACS have now been recognized to occur in a wide variety of disease entities. These include sepsis, 
severe trauma, severe acute pancreatitis, major burns among others (4). We present five cases to highlight the diagnostic and management challenges in these patients.

\section{Cases presentation}

Krohn's intravesical (indirect) method of measuring IAP was used. Measurements were done at first contact, then at 12 and at 24 hours. Additional parameters recorded included: Base excess, serum bilirubin, total blood count, serum urea and creatinine, urine output, vital signs, peak airway pressure and amount of resuscitation fluid administered and fluid balance in 24 hours as recorded in the patient's input-output chart, among others. Classification of IAH based on the IAP values is as follows (1):

Grade $0<12 \mathrm{mmhg}$

Grade 1 12-15mmhg

Grade 2 16-20mmhg

Grade 3 21-25mmhg

Grade $4>25 \mathrm{mmhg}$

Those patients with mild to moderate IAH (Grade 1-3), but not meeting threshold for ACS were recommended for non-surgical interventions to reduce IAP and those with ACS decompressive laparotomy was performed. Written informed consent was obtained to publish each of these case reports and accompanying images.

\section{Case 1}

The first case was a 45 year old man admitted in the surgical ward for alcoholic pancreatitis. He was later transferred to the critical care unit(CCU) upon developing respiratory failure suspected to be adult respiratory distress syndrome (ARDS).While in CCU he had progressive abdominal distention but bowel sounds were present and he was passing stool. He had oliguria, elevated urea at $23.7 \mathrm{ummol} /$ l(3.6-8.3ummol/l) ,creatinine 204mmol/l(60-8$\mathrm{mmol} / \mathrm{l}$ ) and bilirubin 34umol/l(2-20umol/l). The mean IAP over 24 hour period was $25 \mathrm{mmhg}$ with maximal IAP of $27 \mathrm{mmhg}$. Non-surgical interventions including nasogastric intubation, a flatus tube, optimizing analgesia and titrating IV fluids to needs were unsuccessful. He underwent decompressive laparotomy, debridement of necrotic pancreatic tissue and Bogota bag laparostomy. Following the intervention there was dramatic improvement in urine output, reduction in serum bilirubin and urea and creatinine. He had a second look laparotomy and repeat debridement. He progressed well and was extubated seven days later and discharged to the ward shortly afterwards. The definitive closure of the abdomen was done on day 27 and he was discharged home three days later.

\section{Case 2}

A 28 year old woman from rural Kenya had sustained open flame mixed second and third degree burns to the trunk, upper limbs (estimated burnt surface area (BSA) 40\%) as well as inhalational burns after a kerosene stove explosion. Before admission to our burns critical care unit, she had received 14 liters of normal saline and had been catheterized and a central venous line placed at the referring facility where she had been admitted for 48 hours. On examination she was found to have extensive firm eschars on the anterior chest and abdomen. She was sedated, intubated and mechanically ventilated and continued to have IV fluids resuscitation. In spite of aggressive fluid replacement with ringer's lactate, her urine output remained at $<30$ ml per hour, mean systolic BP (SBP) at $84 \mathrm{mmhg}$, creatinine and urea were markedly elevated at 256 micromole/l and $23 \mathrm{mmol} / \mathrm{l}$ respectively. She had a peak airway pressure of $47 \mathrm{cmH} 20$, central venous pressure of $18 \mathrm{~cm} \mathrm{H20(5-15} \mathrm{cm} \mathrm{H20),serum} \mathrm{lactate}$ was $2 \mathrm{mmol} / \mathrm{l}$ and base excess $1-\mathrm{mEq} / \mathrm{L}$. During the 24 hours following admission, the mean IAP was $25 \mathrm{mmh}$ and maximal IAP 28mmhg. Diagnosis of ACS was suspected and measures to reduce the IAP instituted including nasogastric decompression, flatus tube, reduction of crystalloids, introduction of colloids, administration of diuretics and escharotomy on the chest and abdomen. There was only modest reduction in mean and maximal IAP and modest improvement on renal status, BP and peak airway pressure and a decision for decompressive laparotomy was made but she succumbed before this could be done.

\section{Case 3}

A 43 year old male motorcyclist who had collided head on with a speeding car and was thrown off the motor cycle was admitted with moderate head injury, multiple rib fractures with a haemopneumothorax and lung contusions. He also had bilateral compound tibiofibular fractures and stable pelvic fractures. He was suspected to have abdominal trauma based on positive Focused Assessment with Sonography for Trauma (FAST) but abdominal CT scan did not reveal any solid or hollow viscus injury. He was first managed at a county hospital and admitted to the intensive care unit, where he was intubated and started on mechanical ventilation, IV fluids, had a chest drain 
inserted, the fractures splinted and received five pints of packed red blood cells to correct hemorrhagic shock. He stayed at the county facility for 72 hours before referral to our hospital when he continued to deteriorate. At admission to our CCU, he had systolic BP of $68 \mathrm{mmHg}$, no urine output, massively distended abdomen and initial IAP reading was $33 \mathrm{mmHg}$ with a mean IAP of $30 \mathrm{mmhg}$ and a maximal IAP of $41 \mathrm{mmhg}$. Immediate decompressive laparotomy was performed where the findings were: massive bowel distension, edema and $300 \mathrm{ml}$ of blood in the rectovesical pouch of Douglas with the solid and hollow viscera intact. Temporary abdominal closure was performed using a Bogota bag. There was gradual increase of blood pressure and reduction in IAP. The head injury and pelvic fractures were managed conservatively. The lower limb fractures were stabilized with external fixators. He had a prolonged stay in ICU before being discharged to the ward on day 31 . The open abdomen was continuously managed with change of Bogota bags until it was safe to definitively close the abdomen, which was performed on day 45 and discharged home 2 days later.

\section{Case 4}

A 56-year-old overweight (BMI 31) male smoker with poorly controlled diabetes mellitus and hypertension was referred to our cardiothoracic unit with a ruptured $5.9 \mathrm{~cm}$ infra renal aortic aneurysm. Arriving in shock, he had received 8 liters of crystalloids, 2.5 liters of colloids and 6 units of packed red cells preoperatively. He underwent emergency open repair of the aneurysm, with aortic cross clamp time of 100 minutes. He was discharged to the general ward after three days in CCU. While in the ward he was noted to have oliguria, tachypnoea, tachycardia, oxygen saturation at $77 \%$ and had a systolic BP of $80 \mathrm{mmhg}$, with abdominal distension and reduced bowel sounds. Both chest and abdominal plain radiographs were unremarkable. At this point the serum urea was $44 \mathrm{mmol} / \mathrm{l}$, creatinine at $660 \mathrm{micromol} / \mathrm{l}$, hemoglobin at $7.8 \mathrm{~g} / \mathrm{dl}, \mathrm{PH} 7.28$, $\mathrm{PO} 2$ at $53 \mathrm{mmhg}$ and $\mathrm{PCO} 2$ at $32 \mathrm{mmhg}$ with a $\mathrm{HCO} 3$ at $19 \mathrm{mmol} / \mathrm{l}$. He was readmitted to CCU, where he was sedated, intubated and mechanically ventilated, had nasogastric intubation and fluid resuscitation continued. The mean IAP was $27 \mathrm{mmhg}$ with a maximal reading of $31 \mathrm{mmhg}$. A diagnosis of ACS was made and he was taken to theater for a re-exploration of the abdomen. Intraoperatively, he had massively distended intestines and the aneurysm repair was intact. The abdomen was temporarily closed with a Bogota bag. Post operatively he did well, with the IAP falling dramatically, the urine output increased and his respiratory parameters improved and was off the ventilator after four days. He was transferred to the ward where the definitive closure of the abdomen was done on 14th day and he was allowed home the next day.

\section{Case 5}

The fifth patient was a 52 year old man who developed ACS following colectomy for gangrenous sigmoid colon. He presented in shock with mean SBP of $72 \mathrm{mmhg}$, metabolic acidosis as per the blood gas analysis which had PH 7.32,PC02 4.5kpa and $\mathrm{HCO}$ at $26 \mathrm{mmol} / \mathrm{l}$ and oliguria. He had fluid resuscitation using crystalloids and colloids (haemacel) preoperatively. On the second postoperative day, he was noted to be restless, tachypnoeic, SBP 88mmhg, oliguric in spite of adequate IV fluids and had significant abdominal distension and the stoma was not functioning. He had urea of $29 \mathrm{mmol} / \mathrm{l}$, creatinine $230 \mathrm{micromol} / \mathrm{l}$, PH 7.32, po2 $50 \mathrm{mmhg}$, pCO2 at $60 \mathrm{mmhg}$, $\mathrm{Hco} 321 \mathrm{mmol} / \mathrm{l}$. He was admitted to CCU, where he was intubated and ventilated. His mean IAP was $24 \mathrm{mmhg}$ with maximal IAP at $29 \mathrm{mmhg}$. Noninvasive means to reduce IAP did not succeed and he had abdominal re-exploration where massively distended edematous intestines were encountered. The abdomen was closed temporarily with Bogota bag. He responded well with IAP readings dramatically falling, and renal and respiratory parameters also improving. He was extubated 3 days later, transferred to the ward and definitive abdominal closure done on the 23rd day. He was discharged home for colostomy closure after six weeks. Table 1 summarizes the clinical, laboratory and ventilator parameters for patients with ACS.

Table 1: Clinical, Laboratory and Ventilator Parameters for Patients with ACS

\begin{tabular}{|l|l|l|}
\hline Variable & Mean (SD) & Min-Max \\
\hline Mean IAP in 24 hours & $26.1(3.0)$ & $22.3-30.0$ \\
\hline Maximum IAP in 24 hours & $30.0(2.4)$ & $27.0-31.0$ \\
\hline BUN & $36.0(13.7)$ & $20.0-56.0$ \\
\hline Serum creatinine & $194.4(13.0)$ & $179.0-213.0$ \\
\hline Platelet & $128.2(26.8)$ & $97.0-150.0$ \\
\hline Total Bilirubin & $39.8(17.4)$ & $21.0-68.0$ \\
\hline Peak airway pressure & $49.2(7.2)$ & $57.0-23.0$ \\
\hline
\end{tabular}




\section{Discussion}

The incidence of IAH in patients with severe acute pancreatitis (SAP) is high, with 1 in 3 of those with IAH developing full blown ACS and a mortality rate nearly $70 \%$ (5). Factors responsible include: pancreatic and peripancreatic edema (aggravated by excessive IV fluids), ascites, ileus, abdominal wall edema and abdominal pain (6). Our patient's deterioration could easily have been attributed to worsening of the severe pancreatitis and it is not until we measured IAP that we established he had consistently elevated pressures despite the non-surgical measures to reduce IAP already in place. Laparotomy is rarely indicated in severe pancreatitis because even in cases of necrosis, debridement can be achieved using minimally invasive techniques but in our case the decompressive laparotomy achieved both relief of IAP and pancreatic necrosectomy. Though the patient had immediate relief of the distension and normalization of the IAP, managing the open abdomen was complicated by infections necessitating repeat debridements.

The abdominal wall is also affected by elevated IAP, in that visceral edema, free intraperitoneal fluid and abdominal packs distend the abdomen leading to decreased abdominal wall compliance (7). Abdominal wall edema in the setting of shock with attendant aggressive fluid resuscitation also contribute to impaired flexibility $(7,8)$.

Although surgery is very effective in managing ACS in the setting of SAP, it is reserved for those who fail to respond to non-surgical therapeutic interventions such as gut decompression, minimizing IV crystalloids and using more of hypertonic solutions and colloids, use of muscle relaxants, percutaneous drainage and reducing enteral feeds. All that considered, prompt recognition of failed medical management should lead to timely surgical decompression to ensure favorable outcome (9).

The burn patient had severe injuries including inhalational burns complicated by fluid overload as a result of aggressive fluids administration. While the intention was to correct the fluid deficit, the resultant tissue edema may have placed her at risk of ACS. Though ACS is characterized by a tensely distended abdomen, this may not be seen in patients with major torso burns with eschar formation. The risk factors for IAH and ACS in major burns patients are: inhalational burns, burns surface $70 \%$ or greater, massive fluid resuscitation and deep circumferential torso burns (10). In these situations, a combination of SIRS, capillary leak and third spacing and extrinsic compression of chest and abdomen by the eschars contribute to development of IAH and ACS (11). This patient was at high risk of ACS from the word go, though measures to reduce the IAP such as reducing infusion rate and escharotomies were instituted, she may have already sustained irreversible end organ damage by the time a decision to perform decompressive laparotomy was made and hence the bad outcome.

While diuresis, sedation, adequate analgesia ,escharotomy and use of colloids and hypertonic salinedextran may help to reduce IAP in mild and moderate cases of established ACS, decompressive laparotomy is the only treatment option that helps relieve the pressure and improve organ function $(10,11)$. Even with such drastic and invasive intervention, in severe burns complicated by ACS, mortality remains very high (11). Since major burns patients are prone to IAH and ACS, routine IAP monitoring is key in preventing this fatal complication $(11,12)$.

The patient with multiple injuries was initially suspected to have missed abdominal injuries causing the distension and the overall deterioration but with persistently elevated IAP possibility of ACS was entertained and even in his critical condition, a decision was made to take him back to theatre for a laparotomy. Polytrauma patients are at risk of ACS from SIRS causing massive capillary leak and third spacing (13). Another contributing factor is massive blood loss necessitating aggressive resuscitation with IV fluids and blood transfusion, intraperitoneal and retroperitoneal bleeding (13). Even in these critically ill patients, decompressive laparotomy reduces IAP and may also discover major bleeding which can be treated surgically (14).

In the abdominal aortic aneurysm patient, we suspected aortic graft failure leading to a leak with differential diagnosis of ischemic colonic injury and renal failure. With a diagnosis of ACS, it was surprising to only find distended but healthy loops of bowel and the aortic repair site intact. The incidence of IAH following open repair of a ruptured AAA is reported to be as high as $30 \%$ with a mortality approaching $70 \%$ (15). Massive fluid infusion, shock at admission and prolonged cross clamp time are recognized risk factors for ACS development. Abdominal decompression is vital to achieve favorable outcome when managing these patients (16). Prevention of the ACS, with routine measurement and early recognition of rising IAP and expedited decompression of the tense abdomen can 
lead to mortality reduction after aneurysm repair (17). After fluid and electrolyte replacement followed by timely colectomy and Hartmann procedure, we expected the sigmoid volvulus patient to recover well. It was difficult to attribute his rising IAP to ACS because even at admission the patient had presented with massive distension, oliguria and respiratory distress. It was not until we reopened his abdomen and temporarily closed with Bogota bag that we noted the IAP gradually normalize and the renal and respiratory dysfunction resolve. This is a very rare occurrence. Mehmet et al reported on only one case of ACS due to a distended rectal stump following colectomy for gangrenous sigmoid volvulus (18).

\section{Conclusion}

Though rare, abdominal compartment syndrome is associated with adverse outcomes yet it can be prevented by regular measurement of intraabdominal pressure in patients at risk. A wide array of disease conditions can lead to severe intraabdominal hypertension and compartment syndrome. A high index of suspicion, early recognition and timely intervention are key in managing these patients.

\section{References}

1. Malbrain ML, Cheatham ML, Kirkpatrick A, et al. Results from the International Conference of Experts on Intraabdominal Hypertension and Abdominal Compartment Syndrome.I.Definitions. Intensive Care Med. 2006;32:1722-32

2. MacDonnell SP, Lalude OA, Davidson AC. The Abdominal Compartment Syndrome: The Physiological and Clinical Consequences of Elevated Intra-Abdominal Pressure. J Am CollSurg 1996; 183:419

3. Saggi BH, Sugerman HJ, Ivatury RR., et al. Abdominal Compartment Syndrome. J Trauma. 1998;45:597-609

4. Malbrain ML, Chiumello D., Pelosi P, etal. Prevalence of Intra-Abdominal Hypertension in Critically Ill Patients: A Multicentre Epidemiological Study. Intensive Care Med 2004; 30:822.

5. De Waele JJ, Leppaniemi AK. Intra-abdominal Hypertension in Acute Pancreatitis.World J Surg 2009; 33:1128-33,

6. Ke L, Ni HB, Sun JK, et al. Risk Factors and Outcome of Intra-Abdominal Hypertension in Patients with Severe Acute Pancreatitis. World J Surg 2012; 36:171-8
7. Cheatham ML. Abdominal Compartment Syndrome. Pathophysiology And Definitions. Scand J Trauma Resusc Emerg Med 2009, 17:10

8. Schein M, Wittmann DH, Aprahamian CC, et al. The Abdominal Compartment Syndrome: The Physiological And Clinical Consequences of Elevated Intra-Abdominal Pressure. J Am CollSurg 1995.180:745-53.

9. Mentula P, Hienonen P, Kemppainen E, et al. Surgical Decompression for Abdominal Compartment Syndrome in Severe Acute Pancreatitis. Arch Surg 2010; 145:764-69

10. Ivy ME, Atweh NA, Palmer J, et al. Intra-abdominal Hypertension and Abdominal Compartment Syndrome in Burn Patients. J Trauma

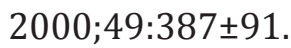

11. Craig B, Oren L,Gad S. Abdominal Compartment Syndrome in a Burn Patient. IMAJ 2002;4:833 \pm 4

12. Kinsky MP, Milner SM, Button B, et al. Resuscitation of Severe Thermal Injury with Hypertonic Saline Dextran: Effects on Peripheral and Visceral Edemain Sheep. J Trauma 2000;49:844 \pm 53 .

13. Leopold P, Milan Sr, Jaroslav M. Abdominal Compartment Syndrome in Polytrauma. Biomed Papers 2004; 148(1), 81-4

14. Nacev TV. Abdominal Compartment Syndrome In Multiple Trauma Patients With Concomitant Abdominal and Head Lesions --Mechanisms and Therapeutical Aspects. Chirurgia Bucur. 2005100(5):507-14

15. Djavani K, Wanhainen A, Björck M. IntraAbdominal Hypertension and Abdominal Compartment Syndrome Following Surgery for Ruptured Abdominal Aortic Aneurysm. Eur J Vasc Endovasc Surg. 2006;31:581

16. Rasmussen TE, Hallett JW, Noel AA, et al. Early Abdominal Closure with Mesh Reduces Multiple Organ Failure After Ruptured Abdominal Aortic Aneurysm Repair: Guidelines From A 10 Year Case-Control Study. J Vasc Surg 2002;35:246e53

17. Bjo"rck M., Wanhainen A., DjavaniK., et al. The Clinical Importance of Monitoring IntraAbdominal Pressure after Ruptured Abdominal Aortic Aneurysm Repair. Scand J Surg 2008; 97:183e90

18. Mehmet Y, BurakIşik, Murat U, et al. Abdominal Compartment Syndrome due to Distended Rectal Stump. Turk J Gastroenterol. 2007;18(3):192-4. 